\title{
Muerte fetal intrauterina: ¿podemos actuar en su prevención?
}

\author{
Zoila Valladares B. ${ }^{1}$, Vanessa García $D .^{1}$, Vanesa Buján $C .{ }^{1}$, Emilio Couceiro N. ${ }^{1}$, \\ Carlos Nicolás López R. ${ }^{1}$ \\ ${ }^{1}$ Servicio de Ginecología y Obstetricia, Complejo Hospitalario Universitario de Vigo. Pontevedra, España.
}

\section{RESUMEN}

Objetivos: Análisis de los factores etiopatogénicos, maternos, fetales y placentarios, asociados a la muerte fetal intrauterina (MFIU). Análisis de los avances en el diagnóstico causal de la MFIU tras instauración de un protocolo específico. Métodos: Estudio descriptivo retrospectivo. Análisis de todos los casos de muerte fetal tardía en gestaciones únicas acontecidas en el Complejo Hospitalario Universitario de Vigo (2005- 2010). Resultados: Hubo 56 casos de muerte fetal tardía. De las gestantes estudiadas, 4 eran menores de 17 años y 19 mayores de 35 años, un $21,4 \%$ fumaban, el $60 \%$ tenía un IMC $\geq 25 \mathrm{~kg} / \mathrm{m} 2$ y el $18 \%$ no controlaron el embarazo. La patología materna predominante fue la tiroidea, mientras que las patologías gestacionales principales fueron diabetes gestacional, preeclampsia y amenaza de parto prematuro. El principal motivo de consulta fue la disminución de movimientos fetales. Se realizó estudio anatomopatológico placentario en el $82 \%$ y necropsia en el $73 \%$. El porcentaje de causa desconocida en el grupo de no protocolo fue $20 \%$ y con protocolo 15\%. Conclusión: El establecimiento de la causa de MFIU es difícil y en algunos casos no posible, aunque sí el reconocimiento de factores de riesgo. La implantación de un protocolo permitió un mayor acercamiento a la causa de la muerte fetal y mejor manejo posterior. La autopsia, el estudio de la placenta, los análisis citogenéticos y el estudio de trombosis materna son la base para el diagnóstico de MFIU.

\section{PALABRAS CLAVE: Muerte fetal anteparto, muerte fetal intrauterina, factores de riesgo, protocolo, necropsia fetal}

\section{SUMMARY}

Objective: Analysis of the pathogenetic maternal fetal and placental factors associated with stillbirth. Analysis of progress in the diagnoses of the cause of stillbirth after the establishment of a specific protocol. Methods: Retrospective descriptive study. Analysis of the cases of late fetal death in singleton pregnancies occurred at the University Hospital of Vigo during the period 2005-2010. Results: We found 56 late fetal deaths. Four were under 17 and 19 above 35 years old, $21 \%$ were smokers, $60 \%$ had a BMI $\geq 25 \mathrm{~kg} / \mathrm{m} 2$ and $18 \%$ had not prenatal care. Thyroid disease was the most prevalent whereas gestational diabetes, preeclampsia and threatened preterm labour were the main obstetric pathologies. The main reason of consultation was decrease of fetal movements. Pathological exam of the placenta was made in $82 \%$ of cases and necropsy in $73 \%$. Rate of unknown cause in the cases of no protocol was $20 \%$ while in the protocol group was $15 \%$. Conclusion: The cause of intrauterine fetal death is difficult to establish. The recognition of certain risk factors is possible. The implementation of a study protocol allowed a better approach to the cause of fetal death and its management. Autopsy, placental examination, cytogenetic analysis, maternal thrombosis study was basic tests for the diagnosis of intrauterine fetal death.

KEY WORDS: Antepartum stillbirth, intrauterine fetal death, risk factors, guideline, fetal necropsy 


\section{INTRODUCCIÓN}

Anualmente se producen en todo el mundo 3,9 millones de muertes fetales. Alrededor de uno a dos millones de muertes quedan sin cuantificar, especialmente en países con deficiente asistencia sanitaria. El $97 \%$ de las muertes fetales ocurren en países en desarrollo, siendo su prevalencia del $3 \%$ en comparación con cifras inferiores al $1 \%$ en países desarrollados (1). En España la tasa de mortalidad fetal tardía anual entre 2000 y 2011 fue del 3-4\%o del total de nacidos (2).

En la evaluación de la muerte fetal intrauterina (MFIU) se puede establecer una clasificación etiológica en: fetales (25-40\%), placentarias (25-35\%) y maternas (5-10\%) (3). Sin embargo, aproximadamente en un $25 \%$ la causa es desconocida (4) a pesar de una evaluación cuidadosa de la evolución clínica, el examen del mortinato, la necropsia y los estudios de laboratorio (5). Existen razones por las que es difícil dar con la causa puesto que ésta suele ser compleja y multifactorial. Además, hay factores de riesgo de muerte fetal que también están presentes en fetos nacidos vivos, como la edad materna avanzada, la obesidad, la raza negra, el hábito tabáquico, el consumo de alcohol y el bajo nivel sociocultural.

La Organización Mundial de la Salud recomienda el uso del término de mortalidad fetal tardía. La clasificación internacional estadística de enfermedades y problemas relacionados con la salud (ICD10) define la mortalidad fetal tardía como la tasa de fetos nacidos muertos mayores de 28 semanas, mayores de $1000 \mathrm{~g}$ de peso, o con una longitud cráneo-rabadilla mayor a $35 \mathrm{~cm}$.

El estudio etiológico de la muerte fetal anteparto debe seguir un protocolo sistemático que permita realizar un acercamiento a la posible causa. A pesar de los costes derivados de dicho estudio, la información obtenida puede ayudar a estimar el riesgo de recurrencia y proporcionar orientación para embarazos futuros.

El objetivo principal fue el análisis de los factores etiopatogénicos asociados a la muerte fetal intraútero, tanto maternos como fetales y placentarios. El objetivo secundario fue valorar la evolución de los resultados en el diagnóstico etiológico de MFIU a raíz de la implantación del protocolo de actuación en estos casos.

\section{MATERIAL Y MÉTODO}

Estudio descriptivo retrospectivo mediante la recogida y análisis de todos los casos de muerte fetal tardía en gestaciones únicas acontecidos en el Complejo Hospitalario Universitario de Vigo desde el 1 de enero de 2005 al 31 de diciembre de 2010.
El número total de casos fue de 56, de los cuales 45 ocurrieron antes de la aplicación del protocolo de estudio y los restantes 11 después (el Protocolo de actuación en casos de MFIU fue implantado en el año 2009). En cada uno de los grupos se han tratado de analizar las características demográficas y los factores etiopatogénicos de la muerte fetal intrauterina.

\section{RESULTADOS}

Se recogieron 56 casos de muerte fetal intrauterina en el periodo 2005-2010. Todas las mujeres pertenecían a la etnia caucásica. La edad media materna fue de 31,4 \pm 6,7 años (media \pm DE) (rango: 15-42 años), destacando 4 casos en gestantes menores de 17 años y 19 casos en gestantes mayores de 35 años.

La mayoría de las pacientes no presentaban antecedentes familiares de relevancia (85,7\%). El 7,1\% presentaban historia familiar de diabetes mellitus, el $5,4 \%$ hipertensión arterial y el 1,8\% epilepsia.

No se encontraron antecedentes personales relevantes en el $80,4 \%$ de los casos. De los casos con enfermedades previas a la gestación, la patología más prevalente fue la tiroidea en un $7,1 \%$ de los casos. Además, se ha encontrado: diabetes, trombofilia, enfermedad respiratoria, epilepsia, hepatitis $B$, adicción a drogas por vía parenteral (ADVP), con un caso respectivamente.

El índice de masa corporal (IMC) fue $\geq 25 \mathrm{~kg} /$ $\mathrm{m} 2$ en el $60 \%$ (30 casos), normal en el $40 \%$ (20 casos) y en 6 casos no estuvo disponible este dato.

No hubo hábitos tóxicos en el $76,8 \%$. El consumo de tabaco estuvo presente en el $21,4 \%$ de los casos y otras drogas en el 1,8\%. No está descrito el consumo de fármacos en el 63,2\%. Los fármacos empleados con mayor frecuencia fueron los relacionados con patología tiroidea $(10,5 \%)$ seguido de benzodiacepinas $(5,3 \%)$ y labetalol $(5,3 \%)$.

El $79,6 \%$ de las gestantes no habían presentado patología alguna durante la gestación. El porcentaje de diabetes gestacional, hipertensión arterial y amenaza de parto pretérmino fue de $6,1 \%$ (3 casos) para cada una de las patologías. Hubo un caso de colestasis.

En la Tabla I se presentan las características demográficas de la población estudiada. No hubo diferencias significativas entre las variables estudiadas entre ambos grupos.

El cribado de aneuploidías realizado en primer o segundo trimestre fue de bajo riesgo en el $88,2 \%$. Se obtuvo un resultado alterado en el $2,9 \%$ y no fue realizado en el $8,8 \%$. La amniocentesis fue realizada en el $35,7 \%$ de las pacientes, siendo el cariotipo normal en todos los casos. 
Tabla I

\section{CARACTERÍSTICAS DEMOGRÁFICAS DE LA POBLACIÓN CON MUERTE FETAL INTRAUTERINA} CON Y SIN PROTOCOLO DE ESTUDIO

\begin{tabular}{lccc}
\hline Variables & Con protocolo & Sin protocolo & Valor $p$ \\
\hline Sin antecedentes familiares & $9(81,8 \%)$ & $39(86,7 \%)$ & NS \\
Edad materna (años) & $29,82 \pm 6,46$ & $31,82 \pm 6,7$ & NS \\
Paridad (\%) & & & NS \\
$\quad$ Primípara & 45,5 & 57,8 & \\
$\quad$ Multípara & 54,5 & 42,2 & NS \\
Sin antecedentes personales & $90,9 \%$ & $77,8 \%$ & \\
Antecedentes obstétricos (\%) & & & NS \\
$\quad$ Ninguno & 72,7 & 77,8 & NS \\
$\quad$ Mal control & $18 ; 2$ & 17,8 & NS \\
Parto prematuro & 0 & 2,2 & NS \\
Edad gestacional (días) & $258,3 \pm 25,5$ & $235 \pm 30,41$ & \\
Patología gestacional (\%) & & & 76,9 \\
$\quad$ Ninguna & 90 & 7,7 & \\
$\quad$ Hipertensión arterial & 0 & 5,1 & \\
Diabetes gestacional & 10 & 2,6 & NS \\
APP & 0 & $62,5 \%$ & NS \\
Colestasis & 0 & 22,2 & \\
IMC ( $>25$ kg/m2) & $30 \%$ & & \\
\hline
\end{tabular}

NS: No significativo ( $p>0,05)$. IMC: Índice de masa corporal.

Las alteraciones ecográficas descritas durante la gestación incluyeron crecimiento intrauterino retardado (CIR) con oligoamnios, malformaciones fetales y alteración funicular en el 5,7\% de los casos respectivamente, seguido en frecuencia de polihidramnios en el 3,8\% (2 casos) y alteraciones placentarias también en el 3,8\% (2 casos). Se ha descrito un caso de macrosoma, un caso de CIR, un caso de oligoamnios aislado, un caso de circular de cordón y un caso de hidrops fetal. En el $66 \%$ de los casos (35 pacientes) no se encontró alteraciones ecográficas.

El motivo de consulta cuando se realizó el diagnóstico de MFIU fue por disminución de movimientos fetales $(35 \%)$, hallazgo ecográfico $(26,4 \%)$, dinámica uterina $(22,6 \%)$, metrorragia $(11,3 \%)$, rotura prematura de membranas $(5,3 \%)$, y otras causas $(1,9 \%)$. La ecografía realizada al ingreso fue normal en el $42,3 \%$ de los casos (22 pacientes). El hallazgo ecográfico más frecuente fue la presencia de oligoamnios en el $32,7 \%$ de los casos (17 pacientes) seguido de diagnóstico de CIR en el 13,4\%. La presencia de polihidramnios fue descrita en el $5,8 \%$
(3 casos). Un 3,8\% presentaron hallazgos de desprendimiento prematuro de placenta normoinserta (DPPNI) (2 casos) y 1,9\% de hidrops fetal ( 1 caso).

En cuanto al sexo fetal del total de MFIU el peso fetal promedio fue de 2.095 gramos (femenino $44,6 \%$ y masculino $55,4 \%$ ). En el grupo con protocolo el peso fetal promedio fue de 2.624 gramos (femenino $45,5 \%$ y masculino $54,5 \%$ ); en el grupo sin protocolo el peso fetal promedio fue 2.000 gramos (femenino $44,4 \%$ y masculino $55,6 \%$ ).

En la mayoría de los casos, la vía de parto fue vaginal, excepto en aquellos casos en que existía una contraindicación para ello, como útero miomatoso con miomas previos o un caso de coagulación intravascular diseminada (CID) que requirió una finalización inmediata. Hubo fiebre intraparto en el $7,1 \%$ (4 casos).

En cuanto a los hallazgos macroscópicos fetoplacentarios observados, no se encontró ningún tipo de alteración en el $44,6 \%$ de los casos. El $16,1 \%$ los hallazgos fueron funiculares (un caso de inserción velamentosa y dos casos con 2 y 3 circulares de cordón respectivamente), el 14,3\% placen- 
tarios, el $10,7 \%$ fetales (acortamiento de miembros en un feto de una paciente epiléptica y un feto con polidactilia de una paciente de 41 años diagnosticada de diabetes mellitus). En el $14,3 \%$, no constan en la historia clínica.

La necropsia fue realizada en el 73,2\% (41 casos). Se realizó estudio anatomopatológico de la placenta en el $82,1 \%$, siendo el resultado normal en el $53,6 \%$. Se describieron infartos placentarios de relevancia en el 8,9\% (5 casos) y la alteración en la inserción del cordón en el 5,4\% (3 casos). Se confirmó el DPPNI en el 5,4\% (3 casos). El diagnóstico de corioamnionitis fue realizado en el 3,6\% (2 casos) y hubo un caso de compresión de cordón $(1,8 \%)$ y uno de arteria umbilical única $(1,8 \%)$ siendo este último una característica ya conocida previamente por ecografía.

El porcentaje de causa desconocida en el grupo sin protocolo fue de $20 \%$ mientras que en el grupo con protocolo fue de 15\%. Dentro del grupo que no hubo protocolo, en un $37,2 \%$ la causa fue multifactorial, seguida de un $16,2 \%$ de causa placentaria, el $13,9 \%$ de causa materna y un $11,6 \%$ de causa fetal; mientras que en el grupo con protocolo, hubo un $46,15 \%$ de causas placentarias, un $23 \%$ de causas maternas y un $15 \%$ de causas multifactoriales, por lo que se pudo determinar una causa probable en un porcentaje mayor de casos producto de la aplicación del protocolo (Figura 1).

\section{DISCUSIÓN}

A partir de los datos obtenidos, se estima que la tasa de mortalidad fetal tardía en el Complejo Hospitalario Universitario de Vigo es de 2,11 por mil, cercana a la cifra española de 3-4 por mil.

Definimos factores de riesgo como características asociadas al problema sin una relación directa obvia, mientras que las causas son condiciones en las que se conoce un mecanismo factible que lleve al problema. Nuestro examen descriptivo muestra que existen, y están presentes de forma significativa, una serie de factores de riesgo asociados a la

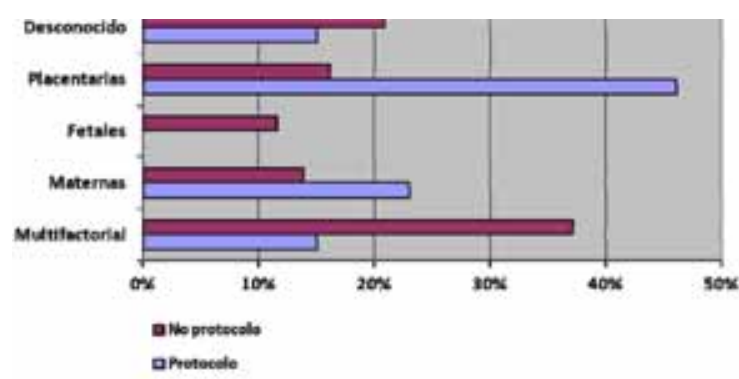

Figura 1. Distribución etiológica de muerte fetal intrauterina por grupos con y sin protocolo de estudio.
MFIU que coinciden con la bibliografía consultada. Algunos de estos factores de riesgo son potencialmente modificables, por lo que las tasas podrían ser reducidas. Además, con su conocimiento se podrían establecer nuevas líneas de investigación, así como prioridades de intervención.

Existen datos sólidos acerca del aumento de la edad materna en el primer embarazo. En nuestro entorno la edad promedio en el parto es de alrededor de 32 años, debido a factores sociales. Una gran cantidad de estudios epidemiológicos han confirmado que la edad materna avanzada supone un aumento del riesgo de muerte fetal intraútero $(1,3,6,7)$. En nuestra población de estudio, casi la mitad de las madres estaban en rangos extremos de edad, 6 menores de 19 años y 19 mayores de 35 años, coincidiendo totalmente con estudios sueco y estadounidense $(1,3)$. Por lo tanto, habría que incidir en la idoneidad de gestación en edades intermedias y alertar a la población de las gestaciones en edades avanzadas o muy precoces.

En cuanto al tabaco, cuya relación con la muerte fetal está bien establecida $(8,9)$, todavía existe un considerable porcentaje de mujeres fumadoras, aunque ha disminuido en las últimas décadas. En nuestro estudio en un $21 \%$ de los casos las mujeres fumaban.

La MFIU se ha registrado con más frecuencia en mujeres primíparas, lo que coincide con nuestra población de estudio (55,4\%). Aunque no es un factor modificable, sí que se podría insistir, dentro de las mujeres primíparas, en actuar sobre otros factores de riesgo, como optimizar el cuidado prenatal y/o realizar una práctica obstétrica basada en la evidencia.

La asociación entre riesgo de muerte fetal e IMC fue informada por primera vez en 1993 (4). Es un hecho conocido que la prevalencia de sobrepeso y obesidad en países desarrollados está creciendo rápidamente, lo que afecta también a la población gestante. En nuestros resultados, se demuestra nuevamente este riesgo, puesto que un $60 \%$ de las madres con óbitos fetales tenían un IMC por encima de $25 \mathrm{~kg} / \mathrm{m} 2$.

Un dato importante es que el $18 \%$ de las muertes fetales se produjeron en gestaciones sin control prenatal, factor causal bien establecido, que también corroboran otros estudios $(10,11)$.

En un $32 \%$ de las MFIU, las madres consultaron en el Servicio de Urgencias por disminución de movimientos fetales, tema importante por el que se ha desarrollado en nuestro servicio un protocolo de actuación específico. El objetivo del mismo es detectar la disminución de movimientos fetales y establecer qué estudios se deben poner en marcha para vigilar con más atención esos embarazos y tratar de disminuir la mortalidad fetal en este grupo de pacientes. 
Se sabe que existen enfermedades médicas que aumentan el riesgo de óbito fetal. Los avances en el cuidado médico y obstétrico han reducido significativamente esta probabilidad, pero el riesgo persiste. Por ejemplo, en nuestro estudio tres mujeres padecían problemas hipertensivos. En cuanto a diabetes, se ha publicado que la diabetes gestacional puede multiplicar el riesgo de muerte fetal hasta por dos (12). En nuestra población, una paciente padecía diabetes pregestacional y tres diabetes gestacional. Robson y cols (13), encontraron una tasa multiplicada por cuatro en cuanto a intolerancia a la glucosa o diabetes gestacional en mujeres con un episodio de muerte fetal previa. De ahí la importancia de la realización de un cribado, en casos de sospecha, ante el suceso de una MFIU. Es conveniente señalar que un $7 \%$ de las pacientes padecían patología tiroidea, aunque hoy en día la muerte fetal debido a esta causa (sobre todo hipotiroidismo) es poco común dado que se realiza control tiroideo, y se controla adecuadamente con la medicación. Por último, comentar un caso de colestasis gravídica en el que la paciente tenía cifras de ácidos biliares por encima de $100 \mu \mathrm{mol} / \mathrm{L}$, valores descritos en la literatura para riesgo de muerte fetal $(40 \mu \mathrm{mol} / \mathrm{L})(14)$.

La hipertensión arterial y la diabetes, como mostramos en el estudio, se mantienen como las patologías más importantes en la contribución a la muerte fetal (15). Según los últimos estudios $(16,17,18,19)$, la diabetes mellitus tipo 2 (DM-II) y la obesidad van a incrementar su importancia en cuanto a los riesgos de muerte fetal en los próximos años. Por lo tanto, a pesar de que estas patologías representan un pequeño porcentaje de las causas del óbito fetal, debe llevarse a cabo un estudio completo para excluir su presencia.

Sabemos que la restricción de crecimiento fetal está fuertemente asociado a la MFIU (20). En nuestro estudio existe un $10 \%$ de patología hallada en ecografía en relación al retraso de crecimiento fetal, tanto diagnóstico de CIR como oligoamnios, lo que coincide con la tasa esperada en población general según la definición teórica de CIR. En ocasiones el fallo de crecimiento no está diagnosticado antenatalmente debido a noxas que podrían afectar al feto durante el intervalo de los controles ecográfico permaneciendo la gestante asintomática.

Sin embargo a pesar de todos los factores de riesgo señalados, hay que destacar que en un $77 \%$ de los casos las pacientes no tenían ningún antecedente obstétrico de interés, en un $80 \%$ de los casos no había patología materna, en un $80 \%$ de los casos no hubo patología obstétrica diagnosticada, en un $66 \%$ no había ningún tipo de alteración ecográfica. Los cribados bioquímicos de anomalías fueron en su mayoría de bajo riesgo y las amniocentesis realizadas mostraron un cariotipo normal en todas.
De todo esto deducimos el poco conocimiento que existe alrededor de este tema y la poca investigación que se ha llevado a cabo. La óptima evaluación de la muerte fetal es tema de controversia y hay muy pocos datos que guíen a los clínicos (21).

En nuestro Servicio se ha comenzado a aplicar un Protocolo de Muerte Fetal Intraútero a partir del año 2009, que incluye la solicitud de una serie de pruebas para esclarecer, en la mayor parte de los casos posibles, la causa de muerte y así poder actuar tanto a nivel preconcepcional como prenatalmente, con diversas medidas preventivas.

La recogida de datos es algo dificultosa, entre otros motivos por la situación emocional de la gestante ante la noticia. Del total de pacientes de nuestro estudio, a un $19,6 \%$ ya se le ha aplicado el protocolo. Debido a un posible periodo adaptativo de éste, la petición de las pruebas no ha sido uniforme. Nuestro protocolo incluye la petición de necropsia (con la autorización de los progenitores), estudio histopatológico de la placenta, examen citogenético y test de hemorragia feto-materna, como estudios básicos tal y como está planteado en otros centros y publicado en la literatura (5). Otros estudios que se solicitan son serología materna, screening de tóxicos en orina, cultivos, estudios hormonales, screening de diabetes, algunos de ellos sólo llevados a cabo en casos de sospecha clínica.

Destacamos de nuestro estudio los siguientes hallazgos, 2 casos de corioamnionitis, 3 casos de DPPNI, una seroconversión del virus de EpsteinBarr (VEB), 6 cultivos subamnióticos positivos (E. Coli, Enterococus faecalis, Estafilococo coagulasa negativo), 4 estudios de trombofilia patológicos (2 con mutación del factor $\mathrm{V}$ Leyden y otros 2 con mutación $20210^{a}$ del gen de la protrombina). Como alteraciones analíticas maternas destacamos 2 hipofibrinogenemias, una de ellas dentro del contexto de un DPPNI, y 2 casos de trombocitopenias. Por lo tanto, la combinación de pruebas podría dilucidar algunas de las causas en las muertes fetales, puesto que al aplicar el protocolo, el porcentaje de causa desconocida o multifactorial disminuye claramente (Figura 1).

\section{CONCLUSIONES}

El establecimiento de la causa de MFIU es difícil y en algunos casos no es posible, aunque sí el reconocimiento de ciertos factores de riesgo. La implantación de un protocolo de actuación en nuestro servicio ha permitido un mayor acercamiento a la causa de muerte fetal y mejor manejo posterior, al reducir las muertes fetales de causa desconocida de un $20 \%$ a un $15 \%$. La autopsia fetal, el estudio de la placenta, los análisis citogenéticos y el estudio de trombosis materna son la base para el diagnóstico de la causa de MFIU. 


\section{REFERENCIAS}

1. Ahnlenius $\mathbf{I}$, Tomasen $P$. The changing panorama of late fetal death in Sweden between 1984 and 1991. Acta Obstet Gynaecol Scand 1999;78:408-14.

2. Instituto Nacional de Estadísticas Vitales. Disponible en: www.ine.es Acceso: Mayo de 2012.

3. Little RE, Weinberg CR. Risk factors for antepartum and intrapartum stillbirth. Am $\mathrm{J}$ Epidemiol 1993;137:1177-89.

4. Korteweg FJ, Erwich JJ, Timmer A, van der Meer J, Ravisé JM, Veeger NJ, Holm JP. Evaluation of 1025 fetal deaths: proposed diagnostic workup. Am J Obstet Gynecol 2012;206:53.e1-12.

5. Chibber R. Unexplained antepartum fetal deaths: what are the determinants? Arch Gynecol Obstet 2005;271:286-91.

6. Fretts RC, Schmittdiel J, McLean FH, Usher RH, Goldman MB. Increased maternal age and the risk of fetal death. N Eng J Med 1995;333:953-7.

7. Cnattingius S, Forman MR, Berendes HW, Isotalo L. Delayed childbearing and risks of adverse perinatal outcome. A population based-study. JAMA 1992;268:886-90.

8. Cnattingius $S$, Haglund $B$, Meirik $O$. Cigarette smoking as risk for late fetal and early neonatal death. BMJ 1988; 297:258-61.

9. Wisborg K, Kesmodel U, Henriksen TB, Olsen SF, Secher NJ. Exposure to tobacco smoke in utero and the risk of stillbirth and death in the first year of life. Am J Epidemiol 2001;154:322-7.

10. Grandi C, Tapia JL, Marshall G, Grupo Colaborativo NEOCOSUR. Evaluación de la severidad, proporcionalidad y riesgo de muerte de recién nacidos de muy bajo peso con restricción del crecimiento fetal. Análisis multicéntrico sudamericano. J Pediatr (Rio J) 2005;81:198-204.

11. Panduro JG, Pérez JJ, Panduro EG, Castro JF,
Vázquez MD. Factores de riesgo en la muerte fetal tardía, Hospital Civil de Guadalajara, México. Rev Chil Obst Ginecol 2011;76:169-74.

12. Conde-Agudelo A, Belizán JM, Díaz-Rosello JL. Epidemiology of fetal death in Latin America. Acta Obstet Gynecol Scand 2000;79:371-8.

13. Robson S, Chan A, Keane RJ, Luke CJ. Subsequent birth outcomes after an unexplained stillbirth: preliminary population-based retrospective cohort study. Aust N Z J Obstet Gynecol 2011;41:29-35.

14. PusI T, Beuers U. Intrahepatic cholestasis of pregnancy. Orphanet J Rare Dis 2007;2:26. Disponible en: http://www.OJRD.com/content/2/1/26

15. Fretts RC, Boyd ME, Usher RH, Usher HA. The changing pattern of fetal death, 1961-1988. Obstet Gynecol 1992;79:35-9.

16. Fox CS, Coady S, Sorlie PD, D'Agostino RB Sr, Pencina MJ, Vasan RS, Meigs JB, Levy D, Savage PJ. Increasing cardiovascular disease burden due to diabetes mellitus. The Framingham Heart Study. Circulation 2007;115:1544-50.

17. Hajjar I, Kotchen TA. Trends in prevalence, awareness, treatment and control of hypertension in the United States 1988-2000. JAMA. 2003;290:199-206.

18. Cundy T, Gamble G, Townend K, Henley PG, MacPherson $\mathrm{P}$, Roberts AB. Perinatal mortality in Type 2 diabetes mellitus. Diabet Med 2000:17:33-9.

19. Ananth CV, Basso O. Impact of pregnancy-induced hypertension on stillbirth and neonatal mortality. Epidemiology 2010;21:118-23.

20. Kady SM, Gardosi J. Perinatal mortality and fetal growth restriction. Bes Pract Res Clin Obstet Gyneacol 2004;18-3:397-410.

21. Silver RM, Varner MW, Reddy U, Goldenberg R, Pinar $H$, Conway D, Bukowski R, Carpenter M, Hogue C, Willinger M, Dudley D, Saade G, Stoll B. Work-up of stillbirth: a review of the evidence. Am J Obstet Gynecol 2007; 196:433-44. 